\title{
ON BICHENG-DEBNATH'S GENERALIZATIONS OF HARDY'S INTEGRAL INEQUALITY
}

\author{
ALEKSANDRA ČIŽMEŠIJA and JOSIP PEČARIĆ
}

(Received 15 September 2000)

\begin{abstract}
We consider Hardy's integral inequality and we obtain some new generalizations of Bicheng-Debnath's recent results. We derive two distinguished classes of inequalities covering all admissible choices of parameter $k$ from Hardy's original relation. Moreover, we prove the constant factors involved in the right-hand sides of some particular inequalities from both classes to be the best possible, that is, none of them can be replaced with a smaller constant.
\end{abstract}

2000 Mathematics Subject Classification. 26D15.

1. Introduction. In the 1920s, Hardy (cf. [3, 4]) proved the following integral inequality: let $p, k \in \mathbb{R}, p>1, k \neq 1$, and for $x \in(0, \infty)$ denote

$$
F(x)= \begin{cases}\int_{0}^{x} f(t) d t, & k>1, \\ \int_{x}^{\infty} f(t) d t, & k<1,\end{cases}
$$

where $f$ is a nonnegative measurable function such that $x^{1-k / p} f \in L^{p}(0, \infty)$. Then

$$
\int_{0}^{\infty} x^{-k} F^{p}(x) d x<\left(\frac{p}{|k-1|}\right)^{p} \int_{0}^{\infty} x^{p-k} f^{p}(x) d x,
$$

unless $f \equiv 0$. The constant $(p /|k-1|)^{p}$ is the best possible.

The relation (1.2), the so-called Hardy's integral inequality, plays a highly important role in mathematical analysis and its applications. Although classical, that result was during the last decade generalized in many different ways by numerous mathematicians. One possibility of generalizing it is to investigate its finite sections, that is, inequalities of the same type, where the outer integrals on both sides of (1.2) are, instead over $(0, \infty)$, taken over some of its subsets.

Very recently two papers, [1, 2], appeared dealing with that subject. In [2], which is the earlier one, Y. Bicheng et al. obtained a whole new class of generalizations of Hardy's integral inequality (1.2). Their results can be joined and stated as follows: let $0 \leq a<b \leq \infty, 1<p<\infty$, and let $f$ be a nonnegative measurable function such that $0<\int_{a}^{b} f^{p}(x) d x<\infty$. Then

$$
\int_{a}^{b} x^{-p}\left(\int_{a}^{x} f(t) d t\right)^{p} d x<\left(\frac{p}{p-1}\right)^{p} \int_{a}^{b} g_{p, a, b}(x) f^{p}(x) d x
$$


where the weight function $g_{p, a, b}$ is given by

$$
g_{p, a, b}(x)=\frac{1}{p}\left(\frac{x}{a}\right)^{(p-1) / p}\left\{\left[1-\left(\frac{a}{b}\right)^{(p-1) / p}\right]^{p}-\left[1-\left(\frac{a}{x}\right)^{(p-1) / p}\right]^{p}\right\} .
$$

Of course, $g_{p, 0, b}=\lim _{a \vee 0} g_{p, a, b}$ and $g_{p, a, \infty}=\lim _{b \rightarrow \infty} g_{p, a, b}$.

Later on, in [1], Bicheng and Debnath derived a class of dual inequalities to (1.3). More precisely, they proved that if the parameters $a, b$, and $p$ are as in [2], and $f$ is $a$ nonnegative measurable function such that $0<\int_{a}^{b} x^{p} f^{p}(x) d x<\infty$, then the inequality

$$
\int_{a}^{b}\left(\int_{x}^{b} f(t) d t\right)^{p} d x<p^{p} \int_{a}^{b} h_{p, a, b}(x) x^{p} f^{p}(x) d x
$$

holds, where the weight function $h_{p, a, b}$ is

$$
h_{p, a, b}(x)=\frac{1}{p}\left(\frac{x}{b}\right)^{1 / p}\left\{\left[1-\left(\frac{a}{b}\right)^{1 / p}\right]^{p}-\left[1-\left(\frac{x}{b}\right)^{1 / p}\right]^{p}\right\} .
$$

Again, $h_{p, 0, b}=\lim _{a \backslash 0} h_{p, a, b}$ and $h_{p, a, \infty}=\lim _{b \rightarrow \infty} h_{p, a, b}$.

Moreover, in cases where $a=0$ or $b=\infty$ the authors proved that the constant factors involved in the right-hand sides of (1.3) and (1.5) are the best possible, while in the case where $0<a<b<\infty$ they provided some upper and lower bounds for maxima of the functions $g_{p, a, b}$ and $h_{p, a, b}$.

Note that Bicheng-Debnath results consider only two particular cases of the parameter $k$ from inequality (1.2): $k=p$ in (1.3), and $k=0$ in (1.5). Therefore, the aim of this paper is to generalize these relations to cover all admissible choices of the parameter $k$ from Hardy's integral inequality.

Hence, our main objective will be to obtain two distinguished classes of inequalities: one of them dealing with $k>1$ and generalizing the class (1.3), and the other one considering $k<1$ and generalizing the class (1.5). Furthermore, we will make a detailed analysis of the weight functions appearing on the right-hand sides of all derived inequalities, what will be used in proving that the obtained constant factor $(p /|k-1|)^{p}$ is the best possible in cases where $a=0$ or $b=\infty$. Finally, we will show that the best possible constant value in the case where $0<a<b<\infty$ is bounded. The established inequalities will conclude the problem of the Bicheng-Debnath-type finite sections of (1.2).

Techniques that will be used in the proofs are mainly based on classical real analysis, especially on the well-known Hölder's and Bernoulli's inequality, and on Fubini's theorem.

2. Preliminaries. First, we present some lemmas which are interesting in their own right and will be used in proofs of our main results.

LEMMA 2.1. Let $0 \leq a<b \leq \infty, 1<p, k<\infty, 1 / p+1 / q=1$, and let $f$ be a nonnegative measurable function such that $0<\int_{a}^{b} t^{p-k} f^{p}(t) d t<\infty$. Then the inequality

$$
\int_{a}^{x} f(t) d t \leq\left(\frac{p}{k-1}\right)^{1 / q}\left(x^{(k-1) / p}-a^{(k-1) / p}\right)^{1 / q}\left[\int_{a}^{x} t^{p-k+(k-1) / p} f^{p}(t) d t\right]^{1 / p}
$$


holds for all $x \in(a, b)$. Moreover, if $a=0$ or $b=\infty$, then there exists a number $x_{0} \in$ $(a, b)$ such that for any $x \in\left(x_{0}, b\right)$ the inequality (2.1) is strict.

Proof. Let $0 \leq a<b \leq \infty$ and $x \in(a, b)$ be arbitrary. Using Hölder's inequality we have

$$
\begin{aligned}
\int_{a}^{x} f(t) d t & =\int_{a}^{x}\left(t^{-(p+1-k) / p}\right)^{1 / q}\left[t^{(p+1-k) / q} f^{p}(t)\right]^{1 / p} d t \\
& \leq\left(\int_{a}^{x} t^{-(p+1-k) / p} d t\right)^{1 / q}\left[\int_{a}^{x} t^{(p+1-k) / q} f^{p}(t) d t\right]^{1 / p} \\
& =\left(\frac{p}{k-1}\right)^{1 / q}\left(x^{(k-1) / p}-a^{(k-1) / p}\right)^{1 / q}\left[\int_{a}^{x} t^{p-k+(k-1) / p} f^{p}(t) d t\right]^{1 / p},
\end{aligned}
$$

so (2.1) is proved. By putting $a=0$ into (2.1), it becomes

$$
\int_{0}^{x} f(t) d t \leq\left(\frac{p}{k-1}\right)^{1 / q} x^{(k-1) /(p 2)}\left[\int_{0}^{x} t^{p-k+(k-1) / p} f^{p}(t) d t\right]^{1 / p} .
$$

Observe that the equality holds in (2.1) and (2.3) if and only if the equality holds in Hölder's inequality. That fact will be very helpful in analyzing strict inequalities for some particular parameters $a$ and $b$ from the statement of the lemma in what follows: suppose that $a=0$ or $b=\infty$, that is, consider the intervals $[0, b)$ and $[a, \infty)$. We have to prove that there exists $x_{0} \in(a, b)$ such that for any $x \in\left(x_{0}, b\right)$ the equality in (2.1) does not hold. Otherwise, there exists a sequence $\left(x_{n}\right)_{n \in \mathbb{N}}$ in $(a, b)$ with the following two properties: $x_{n}>b$ as $n \rightarrow \infty$, and the equality

$$
\int_{a}^{x_{n}} f(t) d t=\left(\frac{p}{k-1}\right)^{1 / q}\left(x_{n}^{(k-1) / p}-a^{(k-1) / p}\right)^{1 / q}\left[\int_{a}^{x_{n}} t^{p-k+(k-1) / p} f^{p}(t) d t\right]^{1 / p}
$$

holds for all $n \in \mathbb{N}$. Therefore, for each $n$ there is the equality in Hölder's inequality, so the functions $t \mapsto t^{(p+1-k) / q} f^{p}(t)$ and $t \mapsto t^{-(p+1-k) / p}$ have to be effectively proportional in $\left[a, x_{n}\right]$ (cf. [3, page 140]), that is, there are nonnegative real constants $c_{n}$ and $d_{n}$, not both zero, such that

$$
c_{n}\left[t^{(p+1-k) /(p 2)} f(t)\right]^{p}=d_{n}\left(t^{-(p+1-k) /(p 2)}\right)^{q} \quad \text { a.e. in }\left[a, x_{n}\right]
$$

Obviously, $c_{n}>0$ (otherwise $t^{-(p+1-k) / p}=0$ a.e. in $\left[a, x_{n}\right]$, what is evidently false), and hence $t^{p-k} f^{p}(t)=\left(d_{n} / c_{n}\right) t^{-1}$ a.e. in $\left[a, x_{n}\right]$. On the other hand, it is impossible to have $d_{n}=0$ for all $n \in \mathbb{N}$, since $x_{n}>b$ and $\int_{a}^{b} t^{p-k} f^{p}(t) d t>0$. So, there exists an integer $N$ such that $d_{N}>0$. Thus $t^{p-k} f^{p}(t)>0$ holds a.e. in $\left[a, x_{N}\right]$. Moreover, since $\left[a, x_{N}\right] \subset\left[a, x_{n}\right]$ holds for $n>N$, we also have that $d_{n} / c_{n}=d_{N} / c_{N}>0$, and hence $t^{p-k} f^{p}(t)=\left(d_{N} / c_{N}\right) t^{-1}$ a.e. in $\left[a, x_{n}\right]$, for all $n>N$. Finally,

$$
\int_{a}^{b} t^{p-k} f^{p}(t) d t=\lim _{n \rightarrow \infty} \int_{a}^{x_{n}} t^{p-k} f^{p}(t) d t=\lim _{n \rightarrow \infty} \frac{d_{N}}{c_{N}} \int_{a}^{x_{n}} t^{-1} d t=\infty .
$$

This contradicts the fact that $\int_{a}^{b} t^{p-k} f^{p}(t) d t<\infty$, so the proof is completed. 
The following lemma contains a result dual to Lemma 2.1. The proofs of both lemmas are similar.

LEMMA 2.2. Let $0 \leq a<b \leq \infty, p, k \in \mathbb{R}, p>1, k<1$, and $1 / p+1 / q=1$. If $f$ is $a$ nonnegative measurable function such that $0<\int_{a}^{b} t^{p-k} f^{p}(t) d t<\infty$, then the inequality

$$
\int_{x}^{b} f(t) d t \leq\left(\frac{p}{1-k}\right)^{1 / q}\left(x^{(k-1) / p}-b^{(k-1) / p}\right)^{1 / q}\left[\int_{x}^{b} t^{p-k+(k-1) / p} f^{p}(t) d t\right]^{1 / p}
$$

holds for all $x \in(a, b)$. Moreover, if $a=0$ or $b=\infty$, then there exists a number $x_{0} \in$ $(a, b)$ such that for any $x \in\left(a, x_{0}\right)$ the equality in (2.7) does not hold.

Proof. First, assume that $0 \leq a<b \leq \infty$ and $x \in(a, b)$ are arbitrary. A direct application of Hölder's inequality yields

$$
\begin{aligned}
\int_{x}^{b} f(t) d t & =\int_{x}^{b}\left(t^{-(p+1-k) / p}\right)^{1 / q}\left[t^{(p+1-k) / 2} f^{p}(t)\right]^{1 / p} d t \\
& \leq\left(\int_{x}^{b} t^{-(p+1-k) / p} d t\right)^{1 / q}\left[\int_{x}^{b} t^{(p+1-k) / q} f^{p}(t) d t\right]^{1 / p} \\
& =\left(\frac{p}{1-k}\right)^{1 / q}\left(x^{(k-1) / p}-b^{(k-1) / p}\right)^{1 / q}\left[\int_{x}^{b} t^{p-k+(k-1) / p} f^{p}(t) d t\right]^{1 / p},
\end{aligned}
$$

so (2.7) is proved. Especially, if $b=\infty$ then (2.7) can be written as

$$
\int_{x}^{\infty} f(t) d t \leq\left(\frac{p}{1-k}\right)^{1 / q} x^{(k-1) /(p 2)}\left[\int_{x}^{\infty} t^{p-k+(k-1) / p} f^{p}(t) d t\right]^{1 / p} .
$$

As in Lemma 2.1, a necessary and sufficient condition for the equalities in (2.7) and (2.9) is that the equality holds in Hölder's inequality.

Now, we consider cases where $a=0$ or $b=\infty$. We need to show that there exists a number $x_{0} \in(a, b)$ such that for any $x \in\left(a, x_{0}\right)$ the inequality in (2.7) is strict. Otherwise, there exists a decreasing sequence $\left(x_{n}\right)_{n \in \mathbb{N}}$ in $(a, b), x_{n} \searrow a$, such that for any $n \in \mathbb{N}$ the inequality (2.7), rewritten for $x=x_{n}$, becomes an equality. Moreover, to every $n \in \mathbb{N}$ there correspond real constants $c_{n}, d_{n} \geq 0$, not both zero, such that

$$
c_{n}\left[t^{(p+1-k) /(p 2)} f(t)\right]^{p}=d_{n}\left(t^{-(p+1-k) /(p 2)}\right)^{q} \quad \text { a.e. in }\left[x_{n}, b\right) .
$$

By the same arguments as in the proof of Lemma 2.1 we conclude that $c_{n}>0$ for all $n \in \mathbb{N}$, and there exists $N \in \mathbb{N}$ such that the relations $d_{n} / c_{n}=d_{N} / c_{N}>0$ and $t^{p-k} f^{p}(t)=\left(d_{N} / c_{N}\right) t^{-1}>0$ a.e. in $\left[x_{n}, b\right)$ hold for all $n>N$. Hence,

$$
\int_{a}^{b} t^{p-k} f^{p}(t) d t=\lim _{n \rightarrow \infty} \int_{x_{n}}^{b} t^{p-k} f^{p}(t) d t=\lim _{n \rightarrow \infty} \frac{d_{N}}{c_{N}} \int_{x_{n}}^{b} t^{-1} d t=\infty
$$

for both cases $a=0$ and $b=\infty$. This is contradictory to $\int_{a}^{b} t^{p-k} f^{p}(t) d t<\infty$, so the lemma is proved. 
The last lemma in this section will be exploited in the analysis of some particular weight functions.

LEMMA 2.3. Let $1<p<\infty$ and $0<c \leq 1$. If $\varphi_{c}:[0, c] \rightarrow \mathbb{R}$ is a function defined by

$$
\varphi_{c}(x)= \begin{cases}1, & x=1 \text { and } c=1, \\ \frac{1}{p} \cdot \frac{c^{p}-x^{p}}{1-x}, & \text { otherwise }\end{cases}
$$

then $\varphi_{c} \in C^{1}[0, c]$ and the following properties hold:

(i) $\varphi_{1}$ is strictly increasing and $1 / p \leq \varphi_{1}(x) \leq 1$ for all $x \in[0,1]$.

(ii) If $0<c<1$, then there exists a unique point $x_{c} \in(0, c)$ such that

$$
\varphi_{c}\left(x_{c}\right)=\max _{x \in[0, c]} \varphi_{c}(x)
$$

Moreover, $0<x_{c}<c^{p /(p-1)}$ and $c^{p} / p<\varphi_{c}\left(x_{c}\right)=x_{c}^{p-1}<c^{p}$.

Proof. Evidently, $\lim _{x>1} \varphi_{1}(x)=\lim _{x>1}(1 / p) \cdot\left(1-x^{p}\right) /(1-x)=1$, so for any $0<c \leq 1$ the function $\varphi_{c}$ is continuous. Since $\varphi_{c}^{\prime}(x)=(1 / p) \cdot\left(\left((p-1) x^{p}-p x^{p-1}+\right.\right.$ $\left.\left.c^{p}\right) /(1-x)^{2}\right)$, it is also $\varphi_{c} \in C^{1}[0, c]$. Observe that

$$
\varphi_{c}(0)=\frac{c^{p}}{p}, \quad \varphi_{c}(c)= \begin{cases}1, & c=1, \\ 0, & 0<c<1 .\end{cases}
$$

To describe the function $\varphi_{c}$ in more detail, let $\psi_{c}:[0, c] \rightarrow \mathbb{R}$ be defined by $\psi_{c}(x)=$ $(p-1) x^{p}-p x^{p-1}+c^{p}$. In other words, $\varphi_{c}^{\prime}(x)=(1 / p) \cdot\left(\psi_{c}(x) /(1-x)^{2}\right)$. It is obvious that $\psi_{c}$ is continuous on $[0, c]$, differentiable in $(0, c)$, and that the inequality $\psi_{c}^{\prime}(x)=$ $p(p-1)(x-1) x^{p-2}<0$ holds in $(0, c)$. Therefore, $\psi_{c}$ is strictly decreasing and the relation $c^{p}=\psi_{c}(0)>\psi_{c}(x)>\psi_{c}(c)=p(c-1) c^{p-1}$ holds for all $x \in(0, c)$.

Now, the proof splits into two separate cases: $c=1$ and $0<c<1$. Consider the case where $c=1$, first. For any $x \in(0,1)$ we then have $\psi_{1}(x)>\psi_{1}(1)=0$ and $\varphi_{1}^{\prime}(x)>$ 0 . Thus, $\varphi_{1}$ is a strictly increasing function and the sequence of inequalities $1 / p=$ $\varphi_{1}(0)<\varphi_{1}(x)<\varphi_{1}(1)=1$ follows immediately whenever $x \in(0,1)$. This proves (i).

However, the case where $0<c<1$ is a little bit different. Here we have $\psi_{c}(0)>0$, but $\psi_{c}(c)<0$. Since $\psi_{c}$ is strictly decreasing and continuous on $[0, c]$, there exists a unique point $x_{c} \in(0, c)$ such that $\psi_{c}\left(x_{c}\right)=0$. Consequently,

$$
\psi_{c}(x)\left\{\begin{array} { l l } 
{ > 0 , } & { 0 \leq x < x _ { c } , } \\
{ = 0 , } & { x = x _ { c } , } \\
{ < 0 , } & { x _ { c } < x \leq c , }
\end{array} \quad \text { that is, } \quad \varphi _ { c } ^ { \prime } ( x ) \left\{\begin{array}{ll}
>0, & 0 \leq x<x_{c}, \\
=0, & x=x_{c}, \\
<0, & x_{c}<x \leq c,
\end{array}\right.\right.
$$

so $\varphi_{c}\left(x_{c}\right)>\varphi_{c}(x)$ for all $x \in[0, c], x \neq x_{c}$. Therefore, $x_{c}$ is the unique global maximizer of the function $\varphi_{c}$ over $[0, c]$ and (2.13) holds. Moreover, the relation $0=$ $\psi_{c}\left(x_{c}\right)=(p-1) x_{c}^{p}-p x_{c}^{p-1}+c^{p}$ yields that $\varphi_{c}\left(x_{c}\right)=(1 / p) \cdot\left(\left(c^{p}-x_{c}^{p}\right) /\left(1-x_{c}\right)\right)=$ 
$x_{c}^{p-1}$. It is only left to determine an upper and lower bound for $\varphi_{c}\left(x_{c}\right)$. We have

$$
\begin{aligned}
\varphi_{c}\left(x_{c}\right) & =\frac{1}{1-x} \int_{x}^{c} t^{p-1} d t \leq \frac{1}{1-x} c^{p-1}(c-x) \\
& =c^{p-1}\left[c-(1-c) \frac{x}{1-x}\right]<c^{p}, \quad 0 \leq x \leq c,
\end{aligned}
$$

so an upper bound, $c^{p}$, for $\varphi_{c}\left(x_{c}\right)$ is obtained. To find a lower bound, note that $c^{p /(p-1)} \in(0, c)$. Then the relations $\varphi_{c}(0)=\varphi_{c}\left(c^{p /(p-1)}\right)=c^{p} / p, \varphi_{c}^{\prime}(0)>0$, and $\varphi_{c}^{\prime}\left(c^{p /(p-1)}\right)=-((p-1) / p) c^{p}\left(1-c^{p /(p-1)}\right)^{-1}<0$ finally imply that $0<x_{c}<c^{p /(p-1)}$ and $\varphi_{c}\left(x_{c}\right)>\varphi_{c}(0)=c^{p} / p$ hold. This proves (ii), so the proof of the lemma is now complete.

REMARK 2.4. Note that the obtained lower bound for $\varphi_{c}\left(x_{c}\right)$ in the case (ii) of Lemma 2.3 can be improved. Namely, since $0<c^{p /(p-1)+1}<c^{p /(p-1)}$, the value $\varphi_{c}\left(c^{p /(p-1)+1}\right)=\left(c^{p} / p\right) \cdot\left(1-c^{p+p /(p-1)}\right) /\left(1-c^{1+p /(p-1)}\right)$ provides better lower bound for $\varphi_{c}\left(x_{c}\right)$. In fact, we have

$$
\varphi_{c}(0)=\frac{c^{p}}{p}<\frac{c^{p}}{p} \cdot \frac{1-c^{p+p /(p-1)}}{1-c^{1+p /(p-1)}}<\varphi_{c}\left(x_{c}\right)<c^{p} .
$$

Of course, an improvement of the lower bound from Lemma 2.3 can also be reached by choosing any other point from $\left(0, c^{p /(p-1)}\right)$.

REMARK 2.5. The case $p=2$ will be of special interest, since $\varphi_{c}\left(x_{c}\right)$ can be calculated exactly. Really, in that case $x_{c}$ is the unique root of the quadratic function $\psi_{c}(x)=x^{2}-2 x+c^{2}$ on $[0, c]$. Therefore, $\varphi_{c}\left(x_{c}\right)=x_{c}=1-\sqrt{1-c^{2}}$.

3. Some new generalizations of Hardy's integral inequality. This section is dedicated to some new generalizations of the relation (1.3). The parameter $k$ from Hardy's original inequality (1.2) will be chosen greater than one.

We start with the basic result, given by the following theorem.

THEOREM 3.1. Let $0<a<b<\infty$ and $1<p, k<\infty$. If $f$ is a nonnegative measurable function such that $0<\int_{a}^{b} x^{p-k} f^{p}(x) d x<\infty$, then

$$
\begin{aligned}
\int_{a}^{b} x^{-k}\left(\int_{a}^{x} f(t) d t\right)^{p} d x & \leq\left(\frac{p}{k-1}\right)^{p} \int_{a}^{b} g(x ; p, k, a, b) x^{p-k} f^{p}(x) d x \\
& <\left(\frac{p}{k-1}\right)^{p} M(p, k, a, b) \int_{a}^{b} x^{p-k} f^{p}(x) d x,
\end{aligned}
$$

where the weight function $g$ is defined by

$$
g(x ; p, k, a, b)=\frac{1}{p}\left(\frac{x}{a}\right)^{(k-1) / p}\left\{\left[1-\left(\frac{a}{b}\right)^{(k-1) / p}\right]^{p}-\left[1-\left(\frac{a}{x}\right)^{(k-1) / p}\right]^{p}\right\}
$$

and $M(p, k, a, b)=\max _{x \in[a, b]} \mathcal{g}(x ; p, k, a, b)$. Moreover,

$$
\frac{1}{p}\left[1-\left(\frac{a}{b}\right)^{(k-1) / p}\right]^{p}<M(p, k, a, b)<\left[1-\left(\frac{a}{b}\right)^{(k-1) / p}\right]^{p} .
$$


Proof. The proof is mostly based on Lemmas 2.1 and 2.3. Using the relation (2.1) we obtain

$$
\begin{aligned}
\int_{a}^{b} x^{-k} & \left(\int_{a}^{x} f(t) d t\right)^{p} d x \\
& \leq\left(\frac{p}{k-1}\right)^{p-1} \int_{a}^{b} x^{-k}\left(x^{(k-1) / p}-a^{(k-1) / p}\right)^{p-1} \int_{a}^{x} t^{p-k+(k-1) / p} f^{p}(t) d t d x \\
& =\left(\frac{p}{k-1}\right)^{p-1} \int_{a}^{b}\left[\int_{t}^{b} x^{-k}\left(x^{(k-1) / p}-a^{(k-1) / p}\right)^{p-1} d x\right] t^{p-k+(k-1) / p} f^{p}(t) d t .
\end{aligned}
$$

Note that the last equality in (3.4) is a consequence of Fubini's theorem. Since

$$
\int_{t}^{b} x^{-k}\left(x^{(k-1) / p}-a^{(k-1) / p}\right)^{p-1} d x=\frac{a^{(1-k) / p}}{k-1}\left\{\left[1-\left(\frac{a}{b}\right)^{(k-1) / p}\right]^{p}-\left[1-\left(\frac{a}{t}\right)^{(k-1) / p}\right]^{p}\right\},
$$

the third row in (3.4) is further equal to

$$
\begin{array}{r}
\left(\frac{p}{k-1}\right)^{p} \int_{a}^{b} \frac{1}{p}\left(\frac{t}{a}\right)^{(k-1) / p}\left\{\left[1-\left(\frac{a}{b}\right)^{(k-1) / p}\right]^{p}-\left[1-\left(\frac{a}{t}\right)^{(k-1) / p}\right]^{p}\right\} t^{p-k} f^{p}(t) d t \\
=\left(\frac{p}{k-1}\right)^{p} \int_{a}^{b} g(t ; p, k, a, b) t^{p-k} f^{p}(t) d t,
\end{array}
$$

so the first relation in (3.1) is proved. To prove the other inequality in (3.1), denote $c=$ $1-(a / b)^{(k-1) / p}$ and $u=1-(a / t)^{(k-1) / p}$. Then $c \in(0,1), u \in[0, c]$, and $g(t ; p, k, a, b)=$ $\varphi_{c}(u)$, where $\varphi_{c}$ is the function defined in the statement of Lemma 2.3. Also, $t \mapsto$ $u(t)$ is a strictly increasing mapping of $[a, b]$ onto $[0, c]$. Therefore, by using (ii) of Lemma 2.3, the weight function $g$ has a unique maximizing point on $[a, b]$. More precisely, there is a unique point $t_{0} \in(a, b)$ such that $g\left(t_{0}, p, k, a, b\right)=\max _{t \in[a, b]}$ $g(t ; p, k, a, b)$. So, $g(t ; p, k, a, b)<M(p, k, a, b)$ a.e. on $[a, b]$. Combining this and the fact that $\int_{a}^{b} x^{p-k} f^{p}(x) d x>0$, we finally have

$$
\left(\frac{p}{k-1}\right)^{p} \int_{a}^{b} g(t ; p, k, a, b) t^{p-k} f^{p}(t) d t<\left(\frac{p}{k-1}\right)^{p} M(p, k, a, b) \int_{a}^{b} t^{p-k} f^{p}(t) d t .
$$

The proof of (3.1) is now completed. The inequality (3.3) holds immediately, if (ii) of Lemma 2.3 is used again.

REMARK 3.2. The estimate (3.3) can be easily improved by an application of Remark 2.4, since considering (2.17) we have

$$
\begin{gathered}
\frac{1}{p}\left[1-\left(\frac{a}{b}\right)^{(k-1) / p}\right]^{p} \frac{1-\left[1-(a / b)^{(k-1) / p}\right]^{p+p /(p-1)}}{1-\left[1-(a / b)^{(k-1) / p}\right]^{1+p /(p-1)}} \\
<M(p, k, a, b)<\left[1-\left(\frac{a}{b}\right)^{(k-1) / p}\right]^{p}
\end{gathered}
$$

REMARK 3.3. Using Theorem 3.1 it is not hard to convince oneself in the validity of the following inequality:

$$
\int_{a}^{b} x^{-k}\left(\int_{a}^{x} f(t) d t\right)^{p} d x<\left(\frac{p}{k-1}\right)^{p}\left[1-\left(\frac{a}{b}\right)^{(k-1) / p}\right]^{p} \int_{a}^{b} x^{p-k} f^{p}(x) d x .
$$


Unfortunately, from the previous analysis (see (3.1) and (3.3)) it is also evident that the constant factor $(p /(k-1))^{p}\left[1-(a / b)^{(k-1) / p}\right]^{p}$ is not the best possible value $\lambda(p, k, a, b)$ for which (3.9) exists. However, Theorem 3.1 shows that $\lambda(p, k, a, b)$ is bounded and provides an explicit upper bound for this constant. In fact,

$$
0<\lambda(p, k, a, b) \leq\left(\frac{p}{k-1}\right)^{p} M(p, k, a, b)<\left(\frac{p}{k-1}\right)^{p}\left[1-\left(\frac{a}{b}\right)^{(k-1) / p}\right]^{p} .
$$

REMARK 3.4. When $p=2$, the inequality (3.1) reduces to the form

$$
\begin{aligned}
\int_{a}^{b} x^{-k}\left(\int_{a}^{x} f(t) d t\right)^{2} d x & \leq\left(\frac{2}{k-1}\right)^{2} \int_{a}^{b} g(x ; 2, k, a, b) x^{2-k} f^{2}(x) d x \\
& <\left(\frac{2}{k-1}\right)^{2}\left[1-\left(\frac{a}{b}\right)^{(k-1) / 4} \sqrt{2-\left(\frac{a}{b}\right)^{(k-1) / 2}}\right] \int_{a}^{b} x^{2-k} f^{2}(x) d x .
\end{aligned}
$$

Observe that the second inequality is a consequence of Remark 2.5.

We continue with analyzing two limit cases of the parameters $a$ and $b$. First, we consider $b=\infty$.

THEOREM 3.5. Let $0<a<\infty$ and $1<p, k<\infty$. If $f$ is a nonnegative measurable function such that $0<\int_{a}^{\infty} x^{p-k} f^{p}(x) d x<\infty$, then

$$
\int_{a}^{\infty} x^{-k}\left(\int_{a}^{x} f(t) d t\right)^{p} d x<\left(\frac{p}{k-1}\right)^{p} \int_{a}^{\infty} g(x ; p, k, a) x^{p-k} f^{p}(x) d x,
$$

where the weight function $g$ is defined by

$$
g(x ; p, k, a)=\frac{1}{p}\left(\frac{x}{a}\right)^{(k-1) / p}\left\{1-\left[1-\left(\frac{a}{x}\right)^{(k-1) / p}\right]^{p}\right\} .
$$

The function $g$ is strictly increasing and $1 / p<g(x ; p, k, a)<1$ holds for all $x>a$. Moreover, the constant $(p /(k-1))^{p}$ is the best possible.

Proof. Using Lemma 2.1 and Fubini's theorem, we have the following sequence of relations:

$$
\begin{aligned}
\int_{a}^{\infty} x^{-k}\left(\int_{a}^{x} f(t) d t\right)^{p} d x \\
\quad<\left(\frac{p}{k-1}\right)^{p-1} \int_{a}^{\infty} x^{-k}\left(x^{(k-1) / p}-a^{(k-1) / p}\right)^{p-1} \int_{a}^{x} t^{p-k+(k-1) / p} f^{p}(t) d t d x \\
\quad=\left(\frac{p}{k-1}\right)^{p-1} \int_{a}^{\infty}\left\{t^{(k-1) / p} \int_{t}^{\infty} x^{(1-k) / p-1}\left[1-\left(\frac{x}{a}\right)^{(1-k) / p}\right]^{p-1} d x\right\} t^{p-k} f^{p}(t) d t \\
=\left(\frac{p}{k-1}\right)^{p} \int_{a}^{\infty} \frac{1}{p}\left(\frac{t}{a}\right)^{(k-1) / p}\left\{1-\left[1-\left(\frac{a}{t}\right)^{(k-1) / p}\right]^{p}\right\} t^{p-k} f^{p}(t) d t \\
=\left(\frac{p}{k-1}\right)^{p} \int_{a}^{\infty} g(t ; p, k, a) t^{p-k} f^{p}(t) d t,
\end{aligned}
$$


so (3.12) is proved. Let $u=1-(a / t)^{(k-1) / p}$. Then $g(t ; p, k, a)=\varphi_{1}(u)$, where $\varphi_{1}$ is defined in Lemma 2.3. According to (i) of Lemma 2.3, the function $g$ is strictly increasing (as composition of such functions $\varphi_{1}$ and $t \mapsto u(t)$ ) and the relation $1 / p<$ $g(t ; p, k, a)<1$ holds for all $t>a$.

We prove that the constant $\lambda=(p /(k-1))^{p}$ is the best possible for (3.12). Otherwise, there exists a smaller constant $C, 0<C<\lambda$, such that

$$
\int_{a}^{\infty} x^{-k}\left(\int_{a}^{x} f(t) d t\right)^{p} d x<C \int_{a}^{\infty} g(x ; p, k, a) x^{p-k} f^{p}(x) d x .
$$

Since $\lim _{\xi \backslash 0}(p /(k-1-\xi))^{p}\left(1-p^{2} \xi /(k-1+(p-1) \xi)\right)=\lambda$, there is a number $\varepsilon \in$ $(0, k-1)$ such that $(p /(k-1-\varepsilon))^{p}\left(1-p^{2} \varepsilon /(k-1+(p-1) \varepsilon)\right)>C$. Let the function $f_{\varepsilon}:[a, \infty) \rightarrow \mathbb{R}$ be defined by $f_{\varepsilon}(x)=x^{(k-1-\varepsilon) / p-1}$. Then

$$
\int_{a}^{\infty} g(x ; p, k, a) x^{p-k} f_{\varepsilon}^{p}(x) d x<\int_{a}^{\infty} x^{p-k} f_{\varepsilon}^{p}(x) d x=\int_{a}^{\infty} x^{-\varepsilon-1} d x=\frac{a^{-\varepsilon}}{\varepsilon}
$$

and, by Bernoulli's inequality, further

$$
\begin{aligned}
\int_{a}^{\infty} x^{-k}\left(\int_{a}^{x} f_{\varepsilon}(t) d t\right)^{p} d x & =\left(\frac{p}{k-1-\varepsilon}\right)^{p} \int_{a}^{\infty} x^{-\varepsilon-1}\left[1-\left(\frac{a}{x}\right)^{(k-1-\varepsilon) / p}\right]^{p} d x \\
& >\left(\frac{p}{k-1-\varepsilon}\right)^{p} \int_{a}^{\infty} x^{-\varepsilon-1}\left[1-p\left(\frac{a}{x}\right)^{(k-1-\varepsilon) / p}\right] d x \\
& =\left(\frac{p}{k-1-\varepsilon}\right)^{p}\left(1-\frac{p^{2} \varepsilon}{k-1+(p-1) \varepsilon}\right) \frac{a^{-\varepsilon}}{\varepsilon}>C \cdot \frac{a^{-\varepsilon}}{\varepsilon} \\
& >C \int_{a}^{\infty} g(x ; p, k, a) x^{p-k} f_{\varepsilon}^{p}(x) d x .
\end{aligned}
$$

This contradicts (3.15), so $\lambda$ is the best possible constant for (3.12). The proof of the theorem is now complete.

REMARK 3.6. When $p=2$, inequality (3.12) reduces to the form

$$
\int_{a}^{\infty} x^{-k}\left(\int_{a}^{x} f(t) d t\right)^{2} d x<\frac{4}{(k-1)^{2}} \int_{a}^{\infty}\left[1-\frac{1}{2}\left(\frac{a}{x}\right)^{(k-1) / 2}\right] x^{2-k} f^{2}(x) d x .
$$

Finally, the case when $0=a<b<\infty$ has already been described in our paper [6], but by using an approach via mixed-means inequalities (cf. [5]). Here we give a different proof of this result.

THEOREM 3.7. Let $0<b<\infty$ and $1<p, k<\infty$. If $f$ is a nonnegative measurable function such that $0<\int_{0}^{b} x^{p-k} f^{p}(x) d x<\infty$, then the inequality

$$
\int_{0}^{b} x^{-k}\left(\int_{0}^{x} f(t) d t\right)^{p} d x<\left(\frac{p}{k-1}\right)^{p} \int_{0}^{b}\left[1-\left(\frac{x}{b}\right)^{(k-1) / p}\right] x^{p-k} f^{p}(x) d x
$$

holds. The constant $(p /(k-1))^{p}$ is the best possible. 
Proof. Directly from Lemma 2.1 (relation (2.3) rewritten with the sign $<$ instead of $\leq$ ) and Fubini's theorem, we have

$$
\begin{aligned}
\int_{0}^{b} x^{-k}\left(\int_{0}^{x} f(t) d t\right)^{p} d x & <\left(\frac{p}{k-1}\right)^{p-1} \int_{0}^{b} x^{(1-k) / p-1} \int_{0}^{x} t^{p-k+(k-1) / p} f^{p}(t) d t d x \\
& =\left(\frac{p}{k-1}\right)^{p-1} \int_{0}^{b}\left[t^{(k-1) / p} \int_{t}^{b} x^{(1-k) / p-1} d x\right] t^{p-k} f^{p}(t) d t \\
& =\left(\frac{p}{k-1}\right)^{p} \int_{0}^{b}\left[1-\left(\frac{t}{b}\right)^{(k-1) / p}\right] t^{p-k} f^{p}(t) d t,
\end{aligned}
$$

so (3.19) is proved. The proof that $(p /(k-1))^{p}$ is the best possible constant for (3.19) is given in [6].

4. Dual inequalities. Our objective in this section is to derive dual inequalities to (3.1), (3.12), and (3.19). In other words, we will consider $k<1$ in (1.2) and obtain some new generalizations of (1.5).

The following theorem presents dual result to Theorem 3.1.

THEOREM 4.1. Suppose $0<a<b<\infty$ and $p, k \in \mathbb{R}, p>1, k<1$. If $f$ is a nonnegative measurable function such that $0<\int_{a}^{b} x^{p-k} f^{p}(x) d x<\infty$, then the inequality

$$
\begin{aligned}
\int_{a}^{b} x^{-k}\left(\int_{x}^{b} f(t) d t\right)^{p} d x & \leq\left(\frac{p}{1-k}\right)^{p} \int_{a}^{b} h(x ; p, k, a, b) x^{p-k} f^{p}(x) d x \\
& <\left(\frac{p}{1-k}\right)^{p} N(p, k, a, b) \int_{a}^{b} x^{p-k} f^{p}(x) d x
\end{aligned}
$$

holds, where the weight function $h$ is defined by

$$
h(x ; p, k, a, b)=\frac{1}{p}\left(\frac{b}{x}\right)^{(1-k) / p}\left\{\left[1-\left(\frac{a}{b}\right)^{(1-k) / p}\right]^{p}-\left[1-\left(\frac{x}{b}\right)^{(1-k) / p}\right]^{p}\right\}
$$

and $N(p, k, a, b)=\max _{x \in[a, b]} h(x ; p, k, a, b)$. Moreover,

$$
\frac{1}{p}\left[1-\left(\frac{a}{b}\right)^{(1-k) / p}\right]^{p}<N(p, k, a, b)<\left[1-\left(\frac{a}{b}\right)^{(1-k) / p}\right]^{p}
$$

Proof. The proof of this theorem is based on Lemmas 2.2 and 2.3, and it is similar to the proof of Theorem 3.1. So, the relation (2.7) and Fubini's theorem imply:

$$
\begin{aligned}
& \int_{a}^{b} x^{-k}\left(\int_{x}^{b} f(t) d t\right)^{p} d x \\
& \quad \leq\left(\frac{p}{1-k}\right)^{p-1} \int_{a}^{b} x^{-k}\left(x^{(k-1) / p}-b^{(k-1) / p}\right)^{p-1} \int_{x}^{b} t^{p-k+(k-1) / p} f^{p}(t) d t d x
\end{aligned}
$$




$$
\begin{aligned}
& =\left(\frac{p}{1-k}\right)^{p-1} \int_{a}^{b}\left[t^{(k-1) / p} \int_{a}^{t} x^{-k}\left(x^{(k-1) / p}-b^{(k-1) / p}\right)^{p-1} d x\right] t^{p-k} f^{p}(t) d t \\
& =\left(\frac{p}{1-k}\right)^{p} \int_{a}^{b} \frac{1}{p}\left(\frac{b}{t}\right)^{(1-k) / p}\left\{\left[1-\left(\frac{a}{b}\right)^{(1-k) / p}\right]^{p}-\left[1-\left(\frac{t}{b}\right)^{(1-k) / p}\right]^{p}\right\} t^{p-k} f^{p}(t) d t \\
& =\int_{a}^{b} h(t ; p, k, a, b) t^{p-k} f^{p}(t) d t
\end{aligned}
$$

and the first inequality in (4.1) is proved. Furthermore, if $c=1-(a / b)^{(1-k) / p}$ and $u=1-(t / b)^{(1-k) / p}$, then $c \in(0,1), u \in[0, c]$, the mapping $t \mapsto u(t)$ is bijective, and $h(t ; p, k, a, b)=\varphi_{c}(x)$ holds, where $\varphi_{c}$ is defined as in Lemma 2.3. Thus, by (ii) of Lemma 2.3, there exists a unique point $t_{0} \in(a, b)$ such that $h\left(t_{0} ; p, k, a, b\right)=$ $\max _{t \in[a, b]} h(t ; p, k, a, b)$. Hence $h(t ; p, k, a, b)<N(p, k, a, b)$ a.e. on $[a, b]$ and (4.3) holds. Finally, using this and the relation $\int_{a}^{b} t^{p-k} f^{p}(t) d t>0$, we obtain the second inequality in (4.1).

REMARK 4.2. According to (2.17) of Remark 2.4, the estimate (4.3) can be improved as follows:

$$
\frac{c^{p}}{p} \cdot \frac{1-c^{p+p /(p-1)}}{1-c^{1+p /(p-1)}}<N(p, k, a, b)<c^{p},
$$

where $c$ is defined in the proof of Theorem 4.1.

REMARK 4.3. Note that the inequality

$$
\int_{a}^{b} x^{-k}\left(\int_{x}^{b} f(t) d t\right)^{p} d x<\left(\frac{p}{1-k}\right)^{p}\left[1-\left(\frac{a}{b}\right)^{(1-k) / p}\right]^{p} \int_{a}^{b} x^{p-k} f^{p}(x) d x
$$

also holds, but the constant appearing on its right-hand side is not the best possible value $\mu(p, k, a, b)$ for which this relation exists. Theorem 4.1 implies that $\mu(p, k, a, b)$ is bounded and

$$
0<\mu(p, k, a, b) \leq\left(\frac{p}{1-k}\right)^{p} N(p, k, a, b)<\left(\frac{p}{1-k}\right)^{p}\left[1-\left(\frac{a}{b}\right)^{(1-k) / p}\right]^{p} .
$$

REMARK 4.4. If $p=2$, then (4.1) reduces to the form

$$
\begin{aligned}
\int_{a}^{b} x^{-k}\left(\int_{x}^{b} f(t) d t\right)^{2} d x & \leq\left(\frac{2}{1-k}\right)^{2} \int_{a}^{b} h(x ; 2, k, a, b) x^{2-k} f^{2}(x) d x \\
& <\left(\frac{2}{1-k}\right)^{2}\left[1-\left(\frac{a}{b}\right)^{(1-k) / 4} \sqrt{2-\left(\frac{a}{b}\right)^{(1-k) / 2}}\right] \int_{a}^{b} x^{2-k} f^{2}(x) d x
\end{aligned}
$$

To conclude this paper, consider limit cases of the parameters $a$ and $b$. As in the preceding section, we start with $b=\infty$. It has to be mentioned that an inequality concerning this case was given in [6]. It is dual to (3.19) and in [6] it was proved by using the concept of mixed means. Here we restate that result and give its other proof. 
THEOREM 4.5. Suppose that $0<a<\infty$ and $p, k \in \mathbb{R}, p>1, k<1$. If $f$ is a nonnegative measurable function such that $0<\int_{a}^{\infty} x^{p-k} f^{p}(x) d x<\infty$, then the inequality

$$
\int_{a}^{\infty} x^{-k}\left(\int_{x}^{\infty} f(t) d t\right)^{p} d x<\left(\frac{p}{1-k}\right)^{p} \int_{a}^{\infty}\left[1-\left(\frac{a}{x}\right)^{(1-k) / p}\right] x^{p-k} f^{p}(x) d x
$$

holds. The constant $(p /(1-k))^{p}$ is the best possible.

Proof. Lemma 2.2, with (2.9) rewritten as strict inequality, and Fubini's theorem yield

$$
\begin{aligned}
\int_{a}^{\infty} x^{-k}\left(\int_{x}^{\infty} f(t) d t\right)^{p} d x & <\left(\frac{p}{1-k}\right)^{p-1} \int_{a}^{\infty} x^{(1-k) / p-1} \int_{x}^{\infty} t^{p-k+(k-1) / p} f^{p}(t) d t d x \\
& =\left(\frac{p}{1-k}\right)^{p-1} \int_{a}^{\infty}\left[t^{(k-1) / p} \int_{a}^{t} x^{(1-k) / p-1} d x\right] t^{p-k} f^{p}(t) d t \\
& =\left(\frac{p}{1-k}\right)^{p} \int_{a}^{\infty}\left[1-\left(\frac{a}{t}\right)^{(1-k) / p}\right] t^{p-k} f^{p}(t) d t
\end{aligned}
$$

so (4.9) is proved. The best possible constant for (4.9) is discussed in [6].

Now, it is only left to describe the case where $a=0$, that is, to obtain a dual result to Theorem 3.7.

THEOREM 4.6. Suppose that $0<b<\infty$ and $p, k \in \mathbb{R}, p>1, k<1$. If $f$ is a nonnegative measurable function such that $0<\int_{0}^{b} x^{p-k} f^{p}(x) d x<\infty$, then the inequality

$$
\int_{0}^{b} x^{-k}\left(\int_{x}^{b} f(t) d t\right)^{p} d x<\left(\frac{p}{1-k}\right)^{p} \int_{0}^{b} h(x ; p, k, b) x^{p-k} f^{p}(x) d x
$$

holds, where the weight function $h$ is defined by

$$
h(x ; p, k, b)=\frac{1}{p}\left(\frac{b}{x}\right)^{(1-k) / p}\left\{1-\left[1-\left(\frac{x}{b}\right)^{(1-k) / p}\right]^{p}\right\} .
$$

Moreover, the function $h$ is strictly decreasing and $1 / p<h(x ; p, k, b)<1$ holds for all $x \in(0, b)$. The constant $(p /(1-k))^{p}$ is the best possible.

Proof. Combining Lemma 2.2 and Fubini's theorem, we have

$$
\begin{aligned}
\int_{0}^{b} x^{-k} & \left(\int_{x}^{b} f(t) d t\right)^{p} d x \\
& <\left(\frac{p}{1-k}\right)^{p-1} \int_{0}^{b} x^{-k}\left(x^{(k-1) / p}-b^{(k-1) / p}\right)^{p-1} \int_{x}^{b} t^{p-k+(k-1) / p} f^{p}(t) d t d x \\
& =\left(\frac{p}{1-k}\right)^{p-1} \int_{0}^{b}\left\{t^{(k-1) / p} \int_{0}^{t} x^{(1-k) / p-1}\left[1-\left(\frac{x}{b}\right)^{(1-k) / p}\right]^{p-1} d x\right\} t^{p-k} f^{p}(t) d t
\end{aligned}
$$




$$
\begin{aligned}
& =\left(\frac{p}{1-k}\right)^{p} \int_{0}^{b} \frac{1}{p}\left(\frac{b}{t}\right)^{(1-k) / p}\left\{1-\left[1-\left(\frac{t}{b}\right)^{(1-k) / p}\right]^{p}\right\} t^{p-k} f^{p}(t) d t \\
& =\left(\frac{p}{1-k}\right)^{p} \int_{0}^{b} h(t ; p, k, b) t^{p-k} f^{p}(t) d t,
\end{aligned}
$$

so (4.11) is proved. If $u=1-(t / b)^{(1-k) / p}$, then $h(t ; p, k, b)=\varphi_{1}(u)$, where $\varphi_{1}$ is defined as in Lemma 2.3. Since the mapping $t \mapsto u(t)$ is strictly decreasing, by (i) of Lemma 2.3 we have that $h$ is also strictly decreasing and that $1 / p<h(t ; p, k, b)<1$ holds for all $t \in(0, b)$.

Now, we have to prove that $\mu=(p /(1-k))^{p}$ is the best possible constant for (4.11). Otherwise, it can be replaced with a smaller constant $D, 0<D<\mu$. Considering $\varepsilon \in$ $(0,1-k)$ such that $(p /(1-k-\varepsilon))^{p}\left(1-p^{2} \varepsilon /(1-k+(p-1) \varepsilon)\right)>D$, and the function $g_{\varepsilon}:(0, b] \rightarrow \mathbb{R}$ defined by $g_{\varepsilon}(x)=x^{(k-1+\varepsilon) / p-1}$ we have

$$
\int_{0}^{b} h(x ; p, k, b) x^{p-k} g_{\varepsilon}^{p}(x) d x<\int_{0}^{b} x^{p-k} g_{\varepsilon}^{p}(x) d x=\int_{0}^{b} x^{\varepsilon-1} d x=\frac{b^{\varepsilon}}{\varepsilon}
$$

and furthermore, using Bernoulli's inequality,

$$
\begin{aligned}
\int_{0}^{b} x^{-k}\left(\int_{x}^{b} g_{\varepsilon}(t) d t\right)^{p} d x & =\left(\frac{p}{1-k-\varepsilon}\right)^{p} \int_{0}^{b} x^{\varepsilon-1}\left[1-\left(\frac{x}{b}\right)^{(1-k-\varepsilon) / p}\right]^{p} d x \\
& >\left(\frac{p}{1-k-\varepsilon}\right)^{p} \int_{0}^{b} x^{\varepsilon-1}\left[1-p\left(\frac{x}{b}\right)^{(1-k-\varepsilon) / p}\right] d x \\
& =\left(\frac{p}{1-k-\varepsilon}\right)^{p}\left(1-\frac{p^{2} \varepsilon}{1-k+(p-1) \varepsilon}\right) \frac{b^{\varepsilon}}{\varepsilon}>D \cdot \frac{b^{\varepsilon}}{\varepsilon} \\
& >D \int_{0}^{b} h(x ; p, k, b) x^{p-k} g_{\varepsilon}^{p}(x) d x .
\end{aligned}
$$

This is a contradiction, so $\mu$ is the best possible constant for (4.11). This completes the proof.

REMARK 4.7. In the case when $p=2$, inequality (4.11) can be written as

$$
\int_{0}^{b} x^{-k}\left(\int_{x}^{b} f(t) d t\right)^{2} d x<\frac{4}{(1-k)^{2}} \int_{0}^{b}\left[1-\frac{1}{2}\left(\frac{x}{b}\right)^{(1-k) / 2}\right] x^{2-k} f^{2}(x) d x .
$$

\section{REFERENCES}

[1] Y. Bicheng and L. Debnath, Generalizations of Hardy's integral inequalities, Int. J. Math. Math. Sci. 22 (1999), no. 3, 535-542. MR 2000h:26037.

[2] Y. Bicheng, Z. Zeng, and L. Debnath, On new generalizations of Hardy's integral inequality, J. Math. Anal. Appl. 217 (1998), no. 1, 321-327. MR 98k:26037. Zbl 893.26008.

[3] G. H. Hardy, J. E. Littlewood, and G. Pólya, Inequalities, 2nd ed., Cambridge University Press, Cambridge, 1988. MR 89d:26016. Zbl 634.26008.

[4] D. S. Mitrinović, J. Pečarić, and A. M. Fink, Inequalities Involving Functions and Their Integrals and Derivatives, Kluwer Academic Publishers Group, Dordrecht, Boston, London, 1991. MR 93m:26036. Zbl 744.26011. 
[5] A. Čižmešija and J. Pečarić, Mixed means and Hardy's inequality, Math. Inequal. Appl. 1 (1998), no. 4, 491-506. MR 99k:26020. Zbl 921.26015.

[6] __ Some new generalisations of inequalities of Hardy and Levin-Cochran-Lee, Bull. Austral. Math. Soc. 63 (2001), no. 1, 105-113. CMP 1812314.

ALEKSANDRA ČIŽMEŠIJA: DEPARTMENT OF MATHEMATICS, UNIVERSITY OF ZAGREB, BIJENIČKA CESTA 30, 10000 ZAGREB, CROATIA

E-mail address: cizmesij@math.hr

Josip PeČArić: Faculty of Textile Technology, University of Zagreb, PierottijeVA 6, 10000 ZAGREB, CROATIA

E-mail address: pecaric@mahazu . hazu.hr 


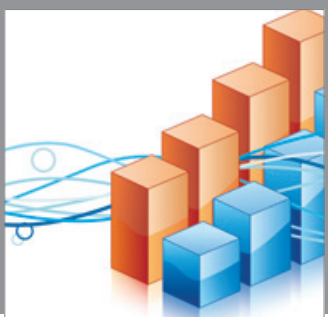

Advances in

Operations Research

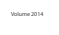

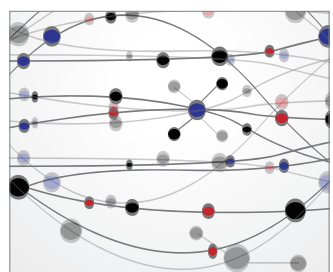

\section{The Scientific} World Journal
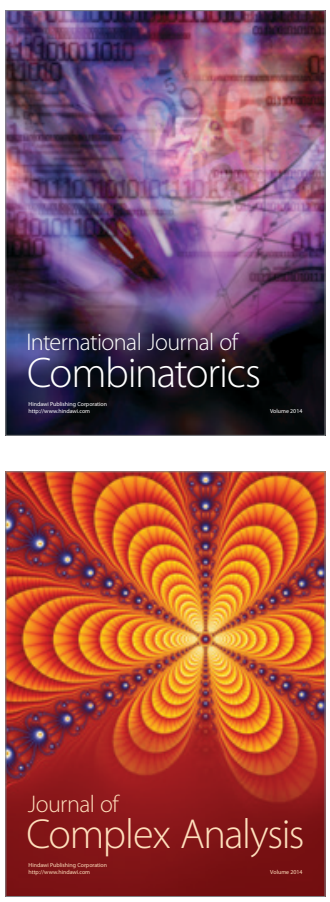

International Journal of

Mathematics and

Mathematical

Sciences
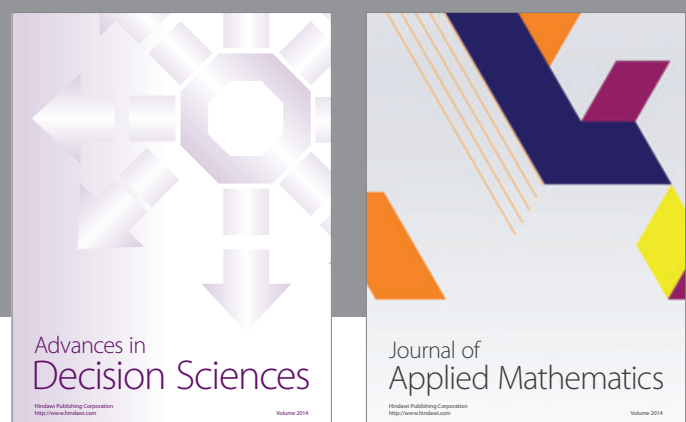

Journal of

Applied Mathematics
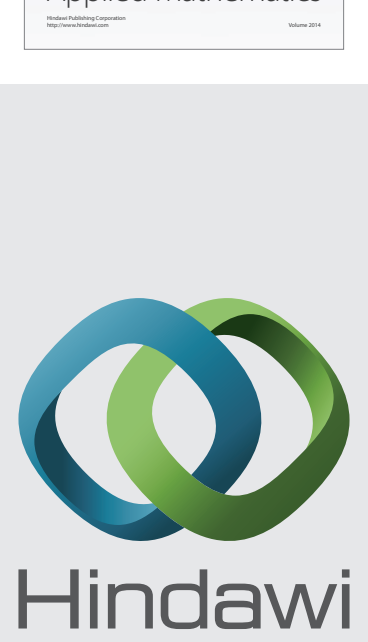

Submit your manuscripts at http://www.hindawi.com
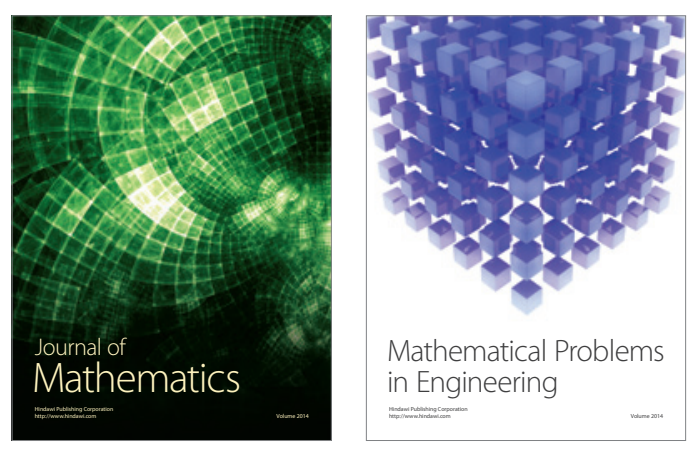

Mathematical Problems in Engineering
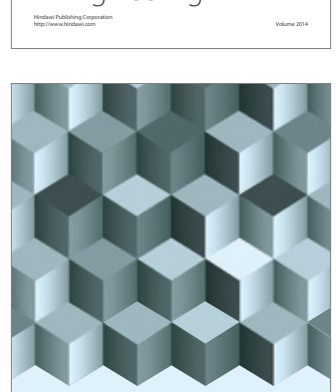

Journal of

Function Spaces
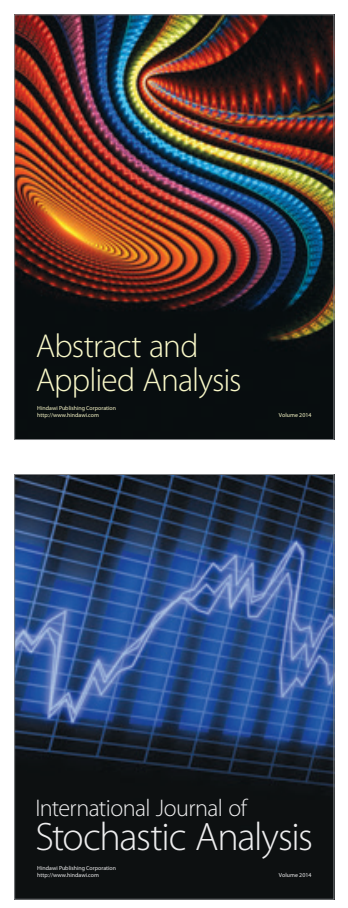

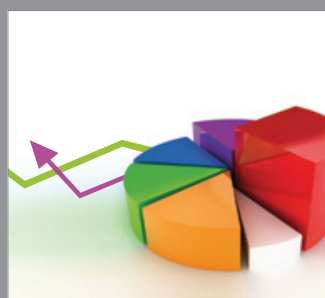

ournal of

Probability and Statistics

Promensencen
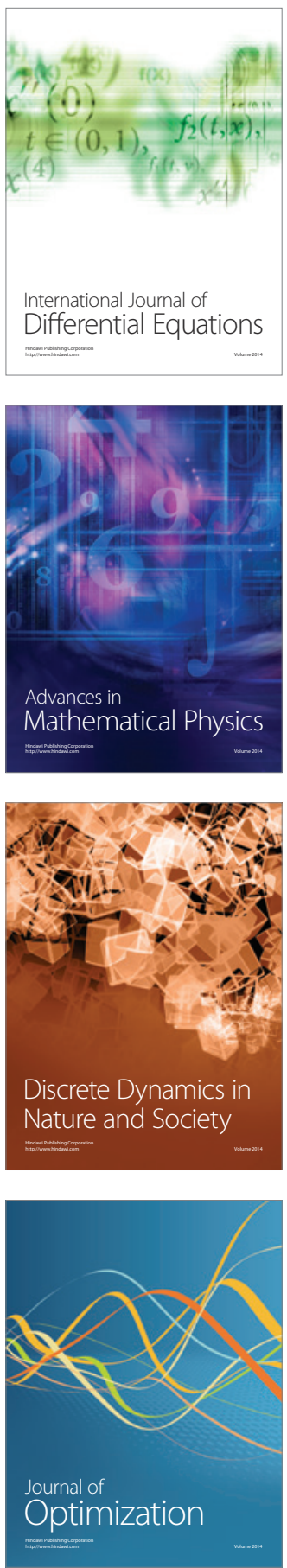